\title{
Shifted axis angular positioning mechanisms - unconventional use of exact constraint design
}

\author{
Ksawery Szykiedans ${ }^{1, *}$, Maciej Bujwan ${ }^{2}$ \\ ${ }^{1}$ Warsaw University of Technology, Faculty of Mechatronics, 02-525 Warszawa, Boboli 8, Poland \\ ${ }^{2}$ Alumnus of Warsaw University of Technology, Faculty of Mechatronics, 02-525 Warszawa, \\ Boboli 8, Poland
}

\begin{abstract}
Paper presents some remarks about designing angular positioners having significant angular displacement and shifted axis of rotation. Those driving mechanism are useful in specific applications where mechanism wraps around other object or there is a need of rotation around virtual axis. Examples of those applications like orthotic robots or gimbals for panoramic motions where developed at Faculty of Mechatronics WUT. Usage of exact constraint method was described including its influence to a kinematic structure of designed mechanism.
\end{abstract}

Keywords: Exact constraint, Camera Head, 3DOF axial positioning.

\section{Introduction}

The engineers designing mechanisms use the principles of kinematics because underconstrained or over-constrained devices will not function. What the precision designer/engineer must be aware that at some scale, everything is a mechanism. Significantly deformable structure will not provide stability of nanometres to precise component. This is often the most important motivation for exact-constraint or kinematic design in precision machines, a need of a separation for a sensitive sub-system or a sensor from the influence of dimensional changing elements. I will present some influence of using exact constraint method to designing process of two kind of 3DOF manipulators. Both cases presents mechanisms of restricted range of angular motion having different aspects of possible shift of its axis of revolution.

Presented case describes very specific example of such conditions. Camera head - a 3 DOF positioning system - requires proper kinematics to ensure not a safety of a camera sensor. The aim is to get a proper motion picture especially during taking panoramic shots. Rotation point of a head and a cameras one has to be the same as no - parallax point of a lens (centre of an entrance pupil). This provides panoramic shot similar to natural viewing by moving only your eyeballs. To achieve panoramic similar to a one's head turning a few centimetres shift between pan axis of rotation and no-parallax point is needed.

\footnotetext{
* Corresponding author: k.szykiedans@mchtr.pw.edu.pl

Reviewers: Wiesława Piekarska, Alžbeta Sapietová
} 


\section{Exact constraint - method of design}

Basics of exact constraint method are taken form the works of J.C. Maxwell [1] but still many engineers do not use this method or not understand it. A quotation from one of founders of exact constraint methodology describes why the method is not so widely or fully used:

"By "dusting off" the principles of kinematics and applying them to machine design, we arrive at the method of Exact Constraint. The method of Exact Constraint has been developed to the point where it comprises a body of knowledge which can be used to routinely create new machine designs which are both high in performance and low in cost. The results are so excellent, yet so obvious; so elegant, yet so simple; that at once they seem both profound and trivial! Perhaps it is this duality which has kept these principles so well hidden. One may ask: "Of what value could anything so trivial be?" And so these principles have been overlooked. They have become disused.» [Blanding, 1992] [2]

The term exact constraint is very overt and expressive when the idea is understood [36]. An unconstrained rigid body has six degrees of freedom (6DOF) - three translations and three rotations. A non-stiff object may have one or more degrees of flexibility that behaves as extra degrees of freedom, relatively speaking. The correct application of constraints would disregard degrees of freedom, one constrain to one DOF. The aim of exact-constraint design is to get desired freedom of motion or full bond by usage of the minimum number of constraints required. For most of the designing engineers previous statements are obvious and are known as true ones. But are they truly used in designing. Man conceptualizes in terms of an ideal constraint, which is absolutely rigid against motion in one or more degrees of freedom and is absolutely free in the remaining degrees of freedom.

The reference by Blanding [6] is an excellent introduction to exact-constraint design and rigid structures. Some other references provide accounts of more specific exact constraint designs such [5].

Most important statements of exact constraint method are quoted below [6, 2].

"Statement 1: Points on the object along the constraint line can move only at right angles to the constraint line, not along it.

Statement 2: Any constraint along a given constraint line is functionally equivalent to any other constraint along the same constraint line (for small motions).

Statement 3: Any pair of constraints whose constraint lines intersect at a given point, is functionally equivalent to any other pair in the same plane whose constraint lines intersect at the same point. This is true for small motions and where the two constraints lie on distinctly different constraint lines.

Statement 4: The axes of a body's rotational degrees of freedom will each intersect all constraints applied to the body

Statement 5: A constraint applied to a body removes that rotational degree of freedom about which it exerts a moment.

Statement 6: Any set of constraints whose constraint lines intersect a complete and independent set of rotational axes, is functionally equivalent to any other set of constraints whose constraint lines intersect the same or equivalent set of rotational axes. This is true for small motions and when each set contains the same number of independent constraints.

Statement 7: An Ideal Sheet Flexure imposes absolutely rigid constraint in its own plane ( $X, Y$, and $\theta z$ ), but it allows three degrees of freedom: $Z, \theta x$, and $\theta y$.

Statement 8: An Ideal Wire Flexure imposes absolutely rigid constraint along its axis $(X)$, but it allows five degrees of freedom: $Y, Z, \theta x, \theta y, \theta z$. 
Statement 9: A constraint (C) properly applied to a body (i.e., without over-constraint) has the effect of removing one of the body's rotational degrees of freedom $(R$ 's). The $R$ removed is the one about which the constraint exerts a moment. A body constrained by $n$ constraints will have $6-n$ rotational degrees of freedom, each positioned such that no constraint exerts a moment about it. In other words, each $R$ will intersect all $C$ 's

Statement 10:Any pair of intersecting rotational degrees of freedom ( $R$ 's) is equivalent to any other pair intersecting at the same point and lying in the same plane. This holds true for small motions.

Statement 11:Two parallel $R$ 's are equivalent to any two parallel $R$ 's, parallel to the first pair and lying in the same plane. They are also equivalent to a single $R$ parallel to the first pair and lying in the same plane; and a T perpendicular to that plane.

Statement 12: When parts are connected in series (cascaded), add the degrees of freedom. When the connections occur in parallel, add constraints."

Introductory statements of presented methodology, especially those about planar movements, are clear and so obvious that are threated piffling. But 3-dimensional analysis of constrains that are not so obvious and can led man to important consideration. I.e. Statement 4 is true if each axis provides uncoupled rotation. An example of such a coupling is the rotation of a threaded part with its related translation. The consequence of infringement of this statement is a complex movement. Similarly statement 9 has the same problem - a single degree of freedom may consist of a coupled rotation and translation. Again it is a third form of kinematic couple of fifth class - lead screw. This behaviour happens when a constraint does not intersect the axis of rotation, thereby introducing translation along the same axis. What lead us to a mechanism with a shifted axis of rotation. Another thought that can be abstract for Blanding works is that over-constrain causes a need of a "perfect" dimensions manufacturing or adding a precise adjusting systems.

Following examples of understanding using statement from Blading works are about shifting axis of rotation in two similar mechanism. But first case will present device with intentionally added adjusting mechanism, second one will prove that general look on the whole kinematics and spotted elasticity can give simpler design.

\section{Camera head for a film making crane}

Camera heads some time called gimbals are 2 or 3-DOF rotary manipulators or positioning devices. It is a quite popular subject for a diploma, connected with media, quite complex thus challenging. Yet not so to stop a student form building one by himself, or with a small support from student's internet TV, as it took place twice, while author was a supervisor of such diplomas at Faculty of Mechatronics WUT. First camera head were built by Maciej Bujwan [7] in first half of 2014, now after being heavily worn will be replaced with a new design of Piotr Grygo.

Let's consider 3-DOF camera head kinematics (Fig. 1) - all possible options have 3 axis rotations, they vary in design of supporting yokes or in a ability of multiple rotation in $Z$ axis. 


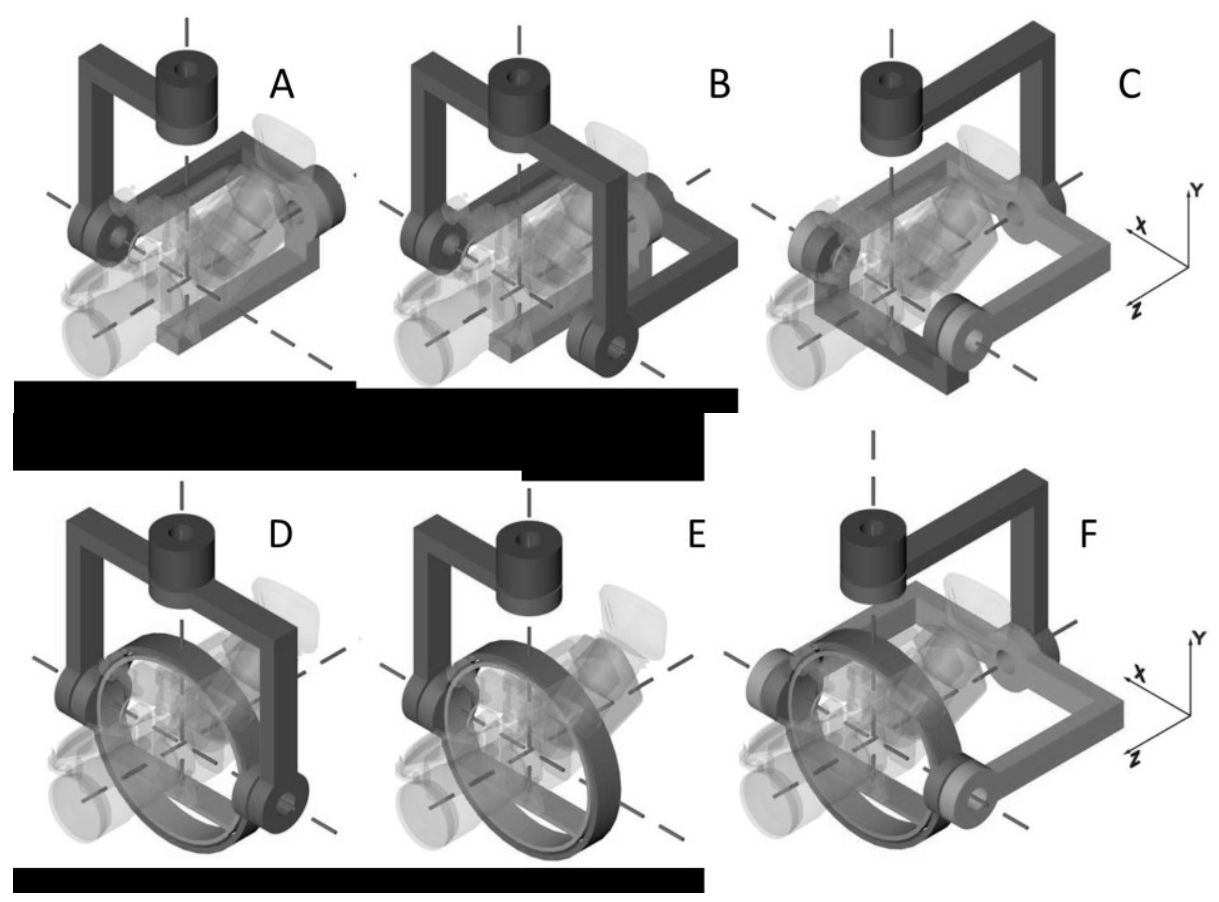

Fig. 1. Kinematics of 3-DOF camera heads

Considering camera head with a connection to a camera we must remember that camera movements not the goal but a way to get proper motion picture. As it was already stated in Introduction shiftage of a Pan axis is desired and should be made very precisely to set a camera to desired panoramic mode (eyeball or head movement). Following pictures (Chyba! Nenašiel sa žiaden zdroj odkazov., Chyba! Nenašiel sa žiaden zdroj odkazov.) explains difference between those two settings.

Fig. 2. Camera panoramic setting with paralaxy

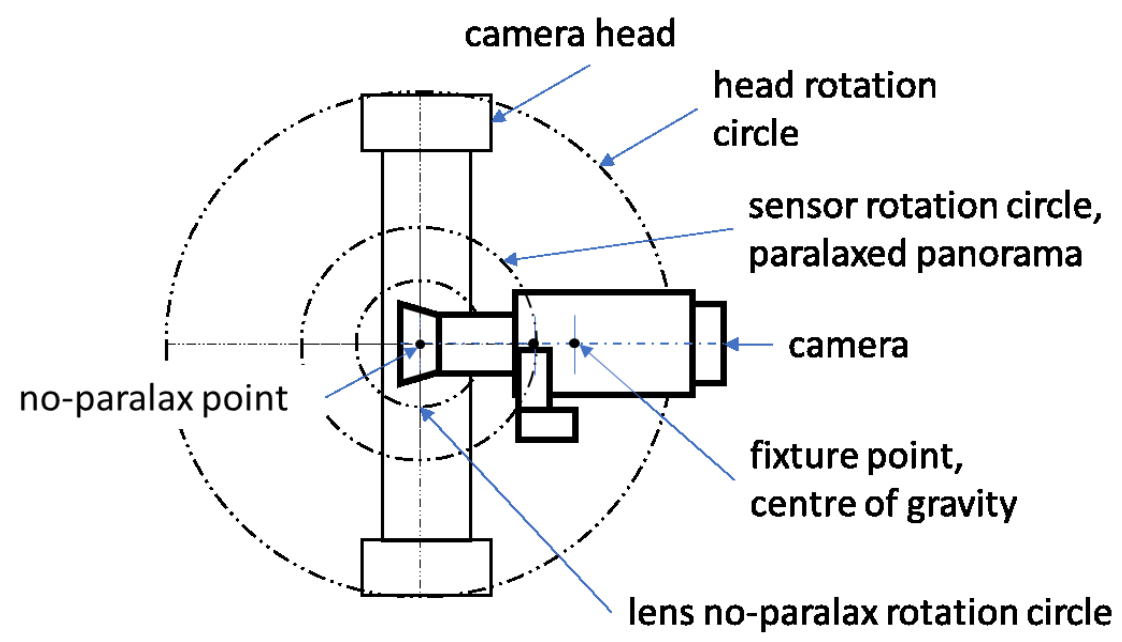

Fig. 3. No -paralax camera panoramic setting 
According to statements of exact constraint method, especially 4 and 9, shifted axes of rotation will cause helical movement. Above case of parallax of camera made panorama is a specific occurrence of those statements because is it planar, that's why it cause only circle not a helix. But analysis of possible shifting of roll or tilt axis will also generate additional movement of a camera sensor (Fig. 4).

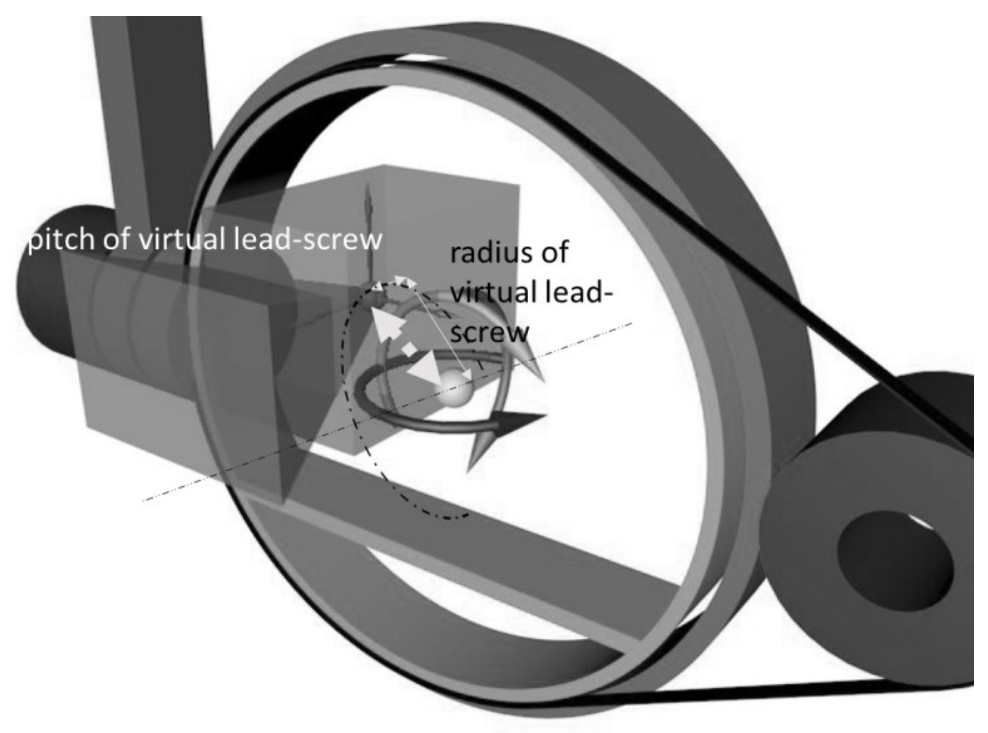

Fig. 4. Helical movement of camera sensor caused by shift between axis of rotation and axis of recording

Such a additional, inattedned movement of a camera is unwanted for at least one reason it wil cause change of image plane and can defocus recorded image or create unpleasent visual effect. To avoid metioned any camera head has to have adjusting mechanism, as well decriben one had (Fig. 5, Chyba! Nenašiel sa žiaden zdroj odkazov.). 


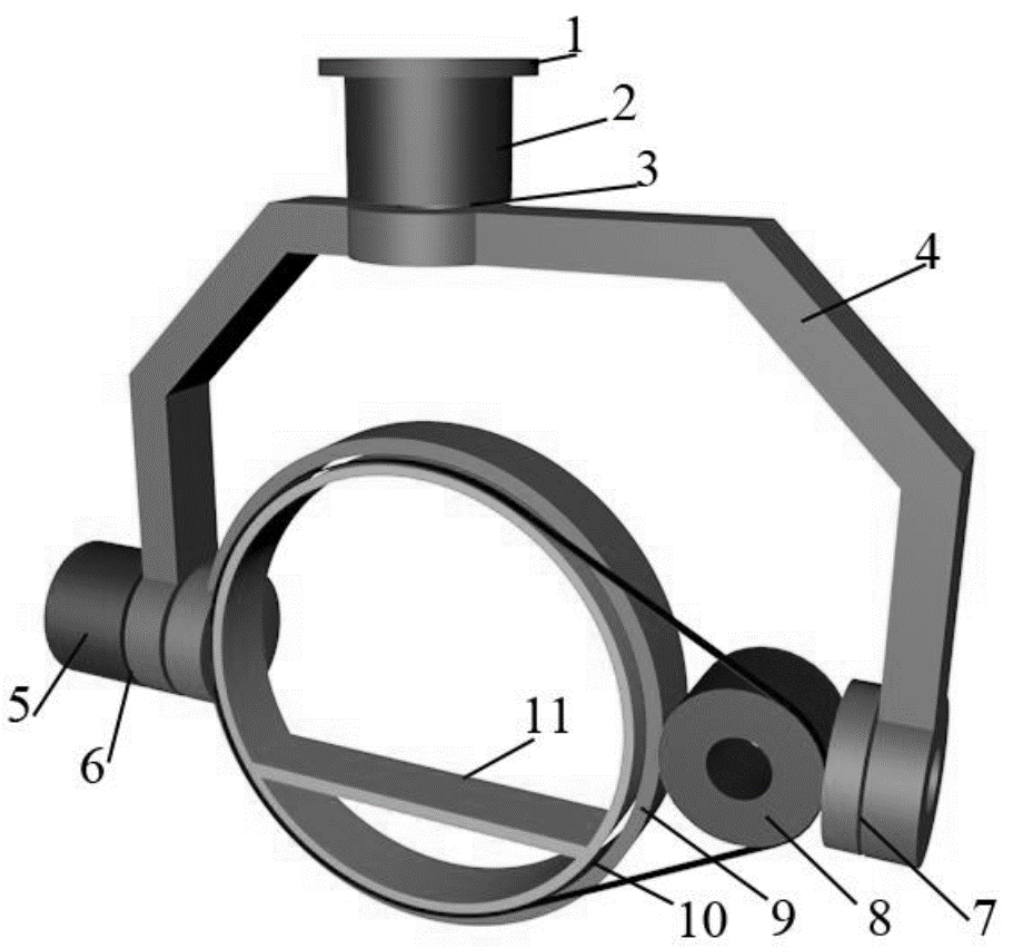

Fig. 5. Camera head designed by M.Bujwan as eengineering thesis at Faculty of Mechatronics WUT ; 1 - head main fixture, 2 - PAN drive, 3 - PAN axis bearing, 4 - frame, 5 - TILT drive, 6 - TILT axis bearing , 7 - ILT support bearing, 8 - ROLL belt drive, 9 - rim, 10 - ROLL ring, 11 - adjustable fixture

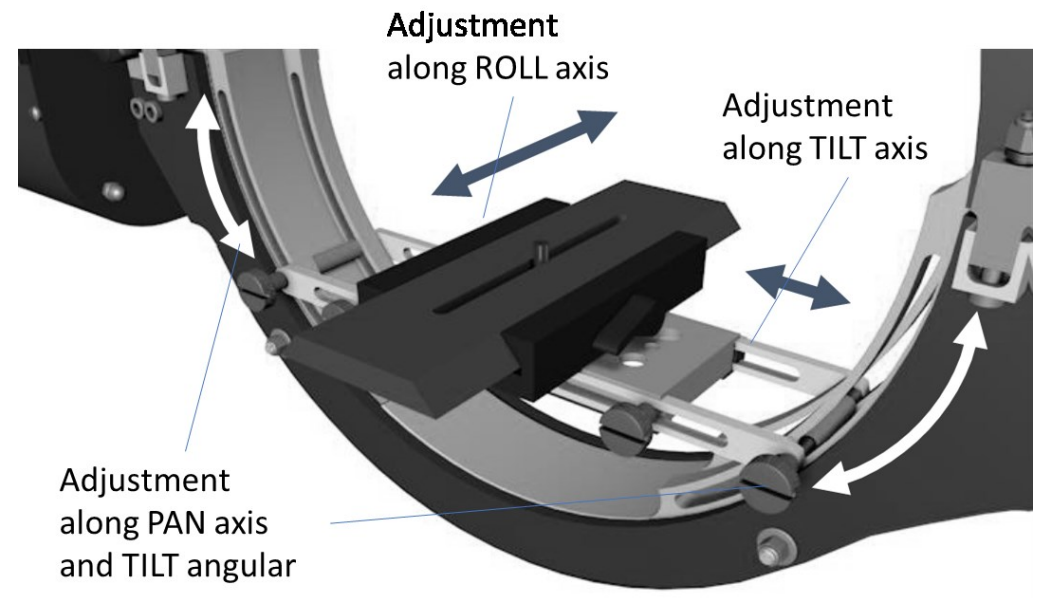

Fig. 6. Adjusting mechanism used in presented camera head

Another subsystem where exact constraint method was used is ROLL unit bearing system (Fig. 7). To provide space for different cameras and to make easy acces to it unit 
recieved form of a ring or a large bearing. To provide as low drag as possible rolling manner was choosen. Usage of such a big rolling bearing was superfluous, it was decided to design a system of same idea but simpler and cheaper. Rolling bearing is over-constrained system - only three rollers, balls or other supporting elements are required to constrain it and define rotation axis. Due to manufacturing and assembling ease frame that holds and supports ROLL unit where split in to symmetrical halves. Somehow it was natural to equip them with the same number of rolling elements.
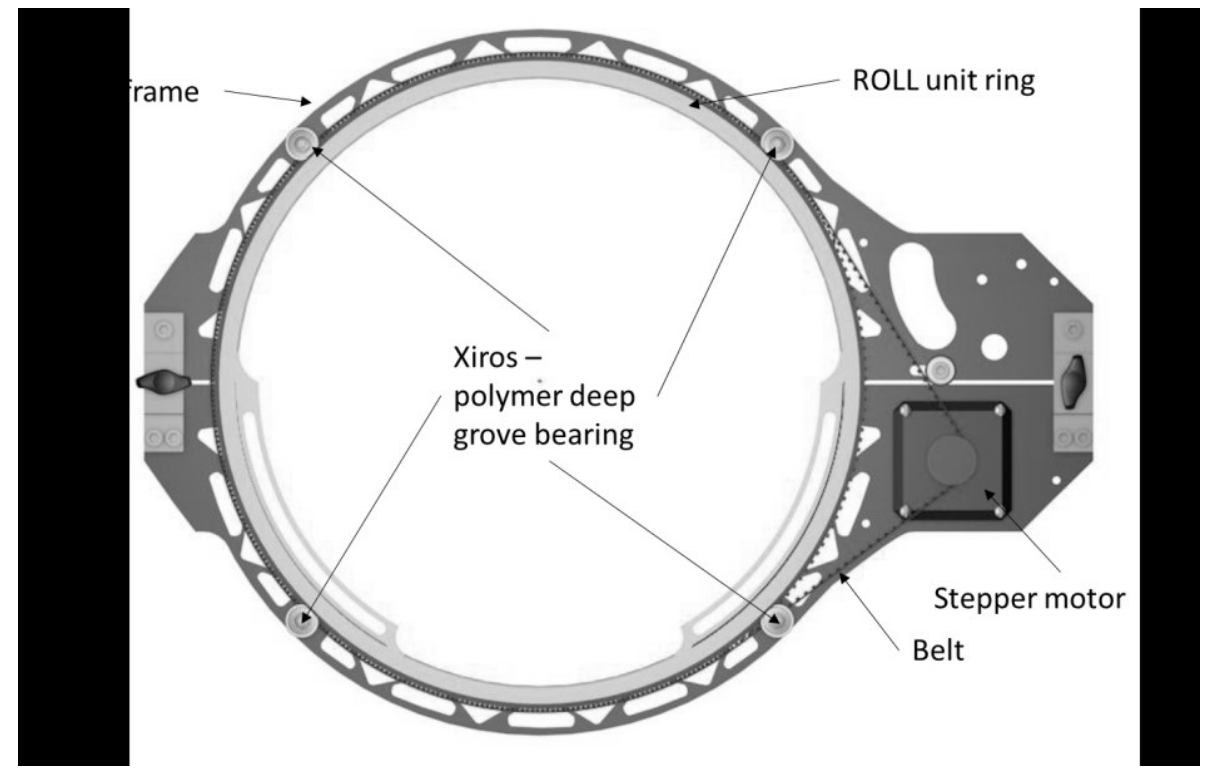

Fig. 7. ROLL unit view, note split frame and symetrical positions of bearings

To provide proper stiffness against twist in TILT axis ring where made wide so at least double row support was required. Such a design will cause single over-constraint, to avoid it extra degree of flexibility had to be added. Frame made as a sheet metal was the first choice but its flexing could affect operationality of whole head. Other solution where chosen. As supporting rolls polymer deep groove ball bearings where used (Fig. 8).

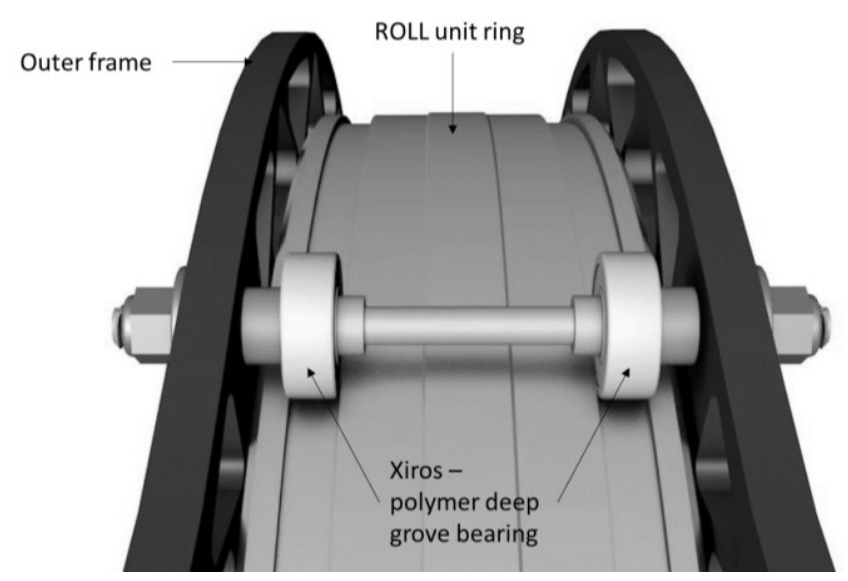

Fig. 8. Side view of support subsystem of ROLL unit 
Those bearing have extra-large axial clearance and are made of flexible (comparing to steel metal) material. These two features provide to design extra degree of freedom to neglect influence of extra constraint.

\section{Conclusion}

Exact Constraint Method as it was published it was connected with designing precise machinery, sometimes heavy, sometimes very sophisticated but always intended to achieve fine/precision movements, processes or products. In presented paper authors gave on example of use exact constraint rules to slightly ethereal product as motion picture. Proper understanding of work of Ch.Evans, D.L. Blanding or A.Slocum does not restrict its usage to machines designing we believe that can be also transferred to virtual world of cinematography.

Prepared with a support of Statutory Found of Faculty of Mechatronics, Warsaw University of Technology.

\section{References}

1. J. C. Maxwell, The Scientific Paper of James Clerk Maxwell (Vol. 2). (Cambridge University Press, London, 1890)

2. L. C. Hale, Principles and Techniques for Desiging Precision Machines. MIT PhD Thesis, 67-82 and 174-204 (1999)

3. T. N. Whitehead, The Design and Use of Instruments and Accurate Mechanism. (Macmillan, New York, 1934)

4. C. J. Evans, Precision Engineering: An Evolutionary View. (Cranfield Press, Bedford, 1989)

5. A. H. Slocum, Precision Machine Design. (SME, Dearborn, Michigan, 1992)

6. D. L. Blanding, Exact Constraint: Machines Design Using Kinematic Principles. (ASME Press, New York, 1999)

7. M. Bujwan, Mechanical design of camera crane head. (WUT, Eng Thesis, 2014) 